\title{
REVIEWS
}

Check for updates

\section{The type I interferonopathies: 10 years on}

Yanick J. Crow $\mathbb{D}^{1,2 \bowtie}$ and Daniel B. Stetson ${ }^{3}$

Abstract | As brutally demonstrated by the COVID-19 pandemic, an effective immune system is essential for survival. Developed over evolutionary time, viral nucleic acid detection is a central pillar in the defensive armamentarium used to combat foreign microbial invasion. To ensure cellular homeostasis, such a strategy necessitates the efficient discrimination of pathogenderived DNA and RNA from that of the host. In 2011, it was suggested that an upregulation of type I interferon signalling might serve as a defining feature of a novel set of Mendelian inborn errors of immunity, where antiviral sensors are triggered by host nucleic acids due to a failure of self versus non-self discrimination. These rare disorders have played a surprisingly significant role in informing our understanding of innate immunity and the relevance of type I interferon signalling for human health and disease. Here we consider what we have learned in this time, and how the field may develop in the future.

Although the term 'type I interferonopathy' entered the medical lexicon only in 2011 (REF. ${ }^{1}$ ), the idea that interferon might be harmful in humans was first posed almost 30 years earlier. On the basis of a series of experiments in rodents, in 1982, Ion Gresser suggested the possibility that interferon might "either cause or contribute to human pathology"2-4. Six years later, Pierre Lebon, a virologist, together with his paediatric neurology colleagues Jean Aicardi and Françoise Goutières, described increased levels of interferon- $\alpha$ (IFNa) activity in the serum and cerebrospinal fluid (CSF) of children affected by a genetic disorder that resembled in utero-acquired viral infection ${ }^{5}$. In so doing, they defined the first Mendelian disease associated with enhanced type I interferon signalling, now referred to as Aicardi-Goutières syndrome (AGS) ${ }^{6}$.

The aforementioned observations overlapped with the advent of recombinant leukocyte interferon as a therapy, and the first reports of neurological disease ${ }^{7,8}$ and autoimmune disease - particularly, systemic lupus erythematosus (SLE) $)^{9,10}$ - apparently triggered by iatrogenic exposure. The neurotoxic potential of type I interferon was further highlighted by the group of Iain Campbell, who described a recapitulation of the neuropathological features of AGS in transgenic mice chronically producing IFNa from astrocytes ${ }^{11,12}$. In 2003, specific attention was drawn to the phenotypic overlap of AGS with SLE and in utero HIV-1 infection, and the possibility that such overlap might result from the common pathological feature of type I interferon upregulation ${ }^{13}$. Subsequent partial dissection of the genetic ${ }^{14-16}$ and molecular ${ }^{17}$ basis of AGS, the identification of mutations in $A C P 5$ as the cause of a Mendelian form of SLE ${ }^{18,19}$ and the definition of a link between $\mathrm{Clq}$ deficiency and IFNa production $^{20,21}$ eventually led to the suggestion that an upregulation of type I interferon signalling might serve as a defining feature of a novel set of Mendelian inborn errors of immunity ${ }^{1}$.

The 10 years since the coining of the term 'type I interferonopathy' have seen a widespread adoption of the concept within the broader classification of autoinflammatory disorders ${ }^{22,23}$. Expert clinical phenotyping, the use of screening assays and the advent of next-generation sequencing have resulted in an increase in the number of type I interferonopathies from seven to close to 40 discrete genotypes, and the recognition of a remarkably broad associated phenotypic spectrum. This rapid increase in knowledge has both informed and been informed by fundamental discoveries in innate immunity, leading to a better understanding of the role of type I interferons in health and disease, and the possibility of therapeutic initiatives aimed at limiting type I interferon signalling. In this Review, we consider what the type I interferonopathies have taught us, and look forward to how this exciting field might develop in the future.

\section{Definitions}

Simply put, the ability to fight infection is necessary for survival $^{24}$, and landmark studies have led to an appreciation of the centrality of foreign nucleic acid detection in the mortal battle waged with viruses ${ }^{25,26}$. We argue that the single most important lesson deriving from the study of the type I interferonopathies is that these essential antiviral systems can also be triggered by host 


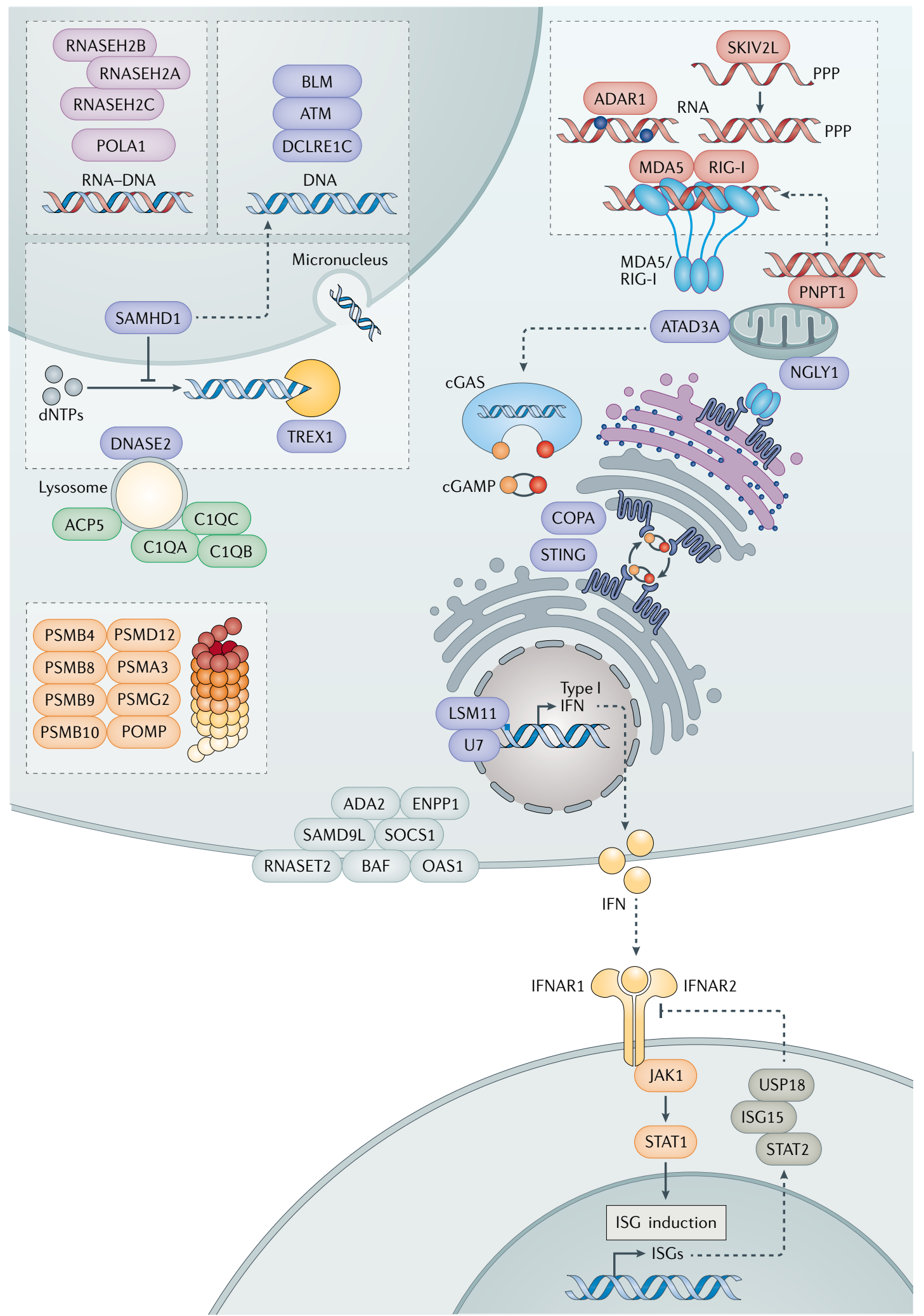

DNA and RNA. That is to say, at the most basic level, the monogenic type I interferonopathies represent a failure of self versus non-self discrimination. If we take a pragmatic approach to their definition ${ }^{27}$, and put aside for the moment the question of the causal relationship to pathogenesis (considered further later), the consistent ex vivo and in vitro observation of enhanced type I interferon signalling in a specific disease setting indicates a biological link between that mutant genotype and interferon homeostasis. On the basis of this simple criterion, we suggest that, currently, at least 38 Mendelian genotypes can be considered as type I interferonopathies (FIG. 1). 
4 Fig. 1 | Putative type I interferonopathy genotypes. Mutations affecting ribonuclease $\mathrm{H} 2$ (RNase H2), a trimeric protein (encoded by RNASEH2A, RNASEH2B and RNASEH2C), and POLA1, the catalytic subunit of DNA polymerase- $\alpha$, an essential component of the DNA-replication machinery, are suggested to result in an alteration in cytosolic levels of RNA-DNA hybrids. Mutations in BLM, ATM and DCLRE1C, encoding the RecQ-like helicase BLM, the DNA repair protein ataxia telangiectasia mutated (ATM) and the DNA double-strand break repair protein Artemis, respectively, lead to the accumulation of products of DNA damage. SAMHD1 hydrolyses deoxynucleoside triphosphates (dNTPs) and may also play a role in DNA repair, while TREX1 degrades single-stranded and double-stranded DNA molecules. DNASE2, encoding the lysosomal endonuclease DNase II (DNASE2), promotes clearance of nucleic acids generated through apoptosis and the phagocytosis of maturating erythroblast nuclei. Mutations in LSM11 and RNU7-1 result in a disturbance of histone stoichiometry, leading to sensing of nuclear DNA. ATAD3A mutations result in a leakage of mitochondrial DNA into the cytosol. All of the aforementioned mutant genotypes signal to interferon induction through cyclic GMPAMP (cGAMP) synthase (cGAS)-STING signalling, which leads to the production of type I interferon (via activation of TANK-binding kinase 1 (TBK1)-interferon regulatory factor 3 (IRF3) and possibly NF-kB (not shown)). Gain-of-function mutations in STING lead to constitutive translocation of the protein from the endoplasmic reticulum to the Golgi apparatus, and dominant negative mutations in coatomer subunit- $\alpha$ (COPA), involved in retrograde Golgi apparatus to endoplasmic reticulum vesicular transport, also result in abnormal STING trafficking. MDA5 (encoded by IFIH1) and RIG-I (encoded by DDX58) normally sense exogenous viral double-stranded RNA (dsRNA); Aicardi-Goutières syndrome-related gain-of-function mutations in MDA5 lower its activation threshold to enable sensing of endogenous dsRNA species. dsRNA-specific adenosine deaminase 1 (ADAR1) deaminates adenosine to inosine, and loss-of-function mutations are proposed to result in the generation of abnormally immunogenic dsRNA species derived from Alu inverted repeats. Mutations in any of these proteins activate interferon signalling via an RNA-sensing pathway involving MAVS. The same is true of mutations in the mitochondrial ribonuclease polynucleotide phosphorylase (PNPT1) and in the RNA helicase SKIV2L, which plays a role in limiting the activation of the cytosolic dsRNA receptor machinery in response to IRE1-mediated RNA degradation. The predominant signalling pathway induced by mutations in N-glycanase (NGLY1), a conserved deglycosylation enzyme, remains unclear. The signalling pathways involved in inducing an interferon signature due to mutations in $\mathrm{C} 1 \mathrm{q}$, the first protein of the classical complement pathway, encoded by $C 1 Q A, C 1 Q B$ and $C 1 Q C$, and $A C P 5$, which encodes the lysosomal phosphatase tartrate-resistant acid phosphatase (TRAP), and in the multiple genes encoding distinct proteasomal components, are also currently undefined. After induction, interferon (IFN) binds to heterodimeric interferon- $\alpha / \beta$ receptor 1 (IFNAR1), leading to phosphorylation of JAK1 and TYK2 and subsequent activation of the transcription factor complex ISGF3. ISGF3 binds to interferon-stimulated response elements in gene promoters and induces the expression of interferon-stimulated genes (ISGs). USP18 is a negative regulator of signalling downstream of IFNAR1. Ubiquitin-like protein ISG15 stabilizes the level of intracellular USP18. Mutations in ISG15 cause a reduction of the level of USP18, resulting in enhanced interferon production. While STAT2 plays a role in positive interferon signal induction, homozygous separation-offunction mutations at p.Arg148 lead to disruption of a role in limiting IFNAR2 signalling. Gain-of-function mutations in JAK1 and STAT2 lead to enhanced type I interferon and other cytokine signalling pathways. We are uncertain of the interferon status of disease related to mutations in adenosine deaminase 2 (ADA2), sterile $\alpha$-motif domain-containing protein 9-like (SAMD9L), suppressor of cytokine signalling 1 (SOCS1), barrier-to-autointegration factor 1 (BAF), ENPP1 ectonucleotide pyrophosphatase/ phosphodiesterase (ENPP1), ribonuclease T2 (RNASET2) and oligoadenylate synthetase 1 (OAS1), encoded by CECR1, SAMD9L, SOCS1, BAF1, ENPP1, RNASET2 and OAS1

respectively, either because of a current lack of or because of the presence of conflicting clinical, in vitro, ex vivo or in vivo evidence. PPP, triphosphate.

Notably, 17 of these 38 mutated gene products act directly on nucleic acid substrates, referred to here as nucleic acid 'metabolism', or mediate their sensing (FIG. 2). Of the remaining 21 genotypes, two encode genes that have a primary role in transducing a DNA signal to an innate immune response, five are involved in type I interferon receptor signalling - leading to a gain of function or affecting negative feedback - and two gene products contribute to the maintenance of mitochondrial integrity. Eight further genes relate to the proteasome, where the precise link to type I interferon induction remains enigmatic. Finally, the relationship of mutations in $A C P 5$ (which encodes tartrate-resistant acid phosphatase type 5) and in $C 1 Q A, C 1 Q B$ or $C 1 Q C$ (which encode the complement component C1q) to disease remains unclear to our mind. What these observations additionally highlight is the importance of 'sterile' self nucleic acid disposal (for example, by TREX1 or DNase II (DNASE2)), 'compartmentalisation' of self nucleic acids from nucleic acid sensors (disrupted by, for example, dysfunction of $\mathrm{N}$-glycanase 1 (NGLY1), ATPase family AAA domain-containing protein $3 \mathrm{~A}$ (ATAD3A) and the U7 small nuclear RNP complex) and modification of endogenous nucleic acids (for example, by double-stranded RNA-specific adenosine deaminase 1 (ADAR1)) in avoiding an autoinflammatory state.

A second interesting lesson arising from the definition of the type I interferonopathies is that mutations in the DNA-sensing pathway and the RNA-sensing pathway can have 'equivalent' effects in terms of disease outcome. This is best exemplified by AGS, where a disturbance of either DNA-mediated signalling (due to mutations affecting TREX1, the RNase H2 complex, the deoxynucleoside triphosphate triphosphohydrolase SAMHD1 and the U7 small nuclear RNP complex) or RNA-mediated signalling (due to mutations affecting ADAR1 or MDA5) can result in indistinguishable phenotypes. This is notable, given that these systems are presumed to have evolved to detect distinct classes of pathogen.

\section{Diagnosis}

Testing for type I interferonopathies. The first description of enhanced interferon signalling in a monogenic type I interferonopathy involved an assessment of the ability of serum and CSF from patients with AGS to protect Madin-Darby bovine kidney cells from death following exposure to vesicular stomatitis virus ${ }^{5}$ (BOX 1). An antibody neutralization assay showed that the protective antiviral activity was dependent on IFNa but not IFN $\beta$, and an absence of IFN $\gamma$ was recorded by radioimmunoassay. Subsequently, it was shown that evaluating the expression of interferon-stimulated genes (ISGs) a so-called interferon signature - was more sensitive in defining AGS mutant status ${ }^{28}$. More recently, digital enzyme-linked immunosorbent assay technology has allowed the direct measurement of IFNa ${ }^{29,30}$. These tests have proven highly useful in directing genetic analysis, the interpretation of sequence variant $\mathrm{s}^{31}$ and the identification of novel type I interferonopathy genotypes ${ }^{32}$, including where a link to interferon signalling had not been previously recognized. Important examples of the latter situation include the demonstration of type I interferon induction due to dysfunction of PSMB8 (REFF ${ }^{33,34}$ ), coatomer subunit- $\alpha$ (COPA $)^{35,36}$ and ATAD3A $\mathrm{A}^{37,38} 2,3$ and 5 years, respectively, after the initial description of disease-associated mutations in the genes encoding these proteins. Tests of type I interferon signalling status are still not included in mainstream clinical medicine assessment, although the use of digital enzyme-linked immunosorbent assay, Nanostring ${ }^{39}$ and other ${ }^{40}$ technologies may change this situation, particularly as the (early) 


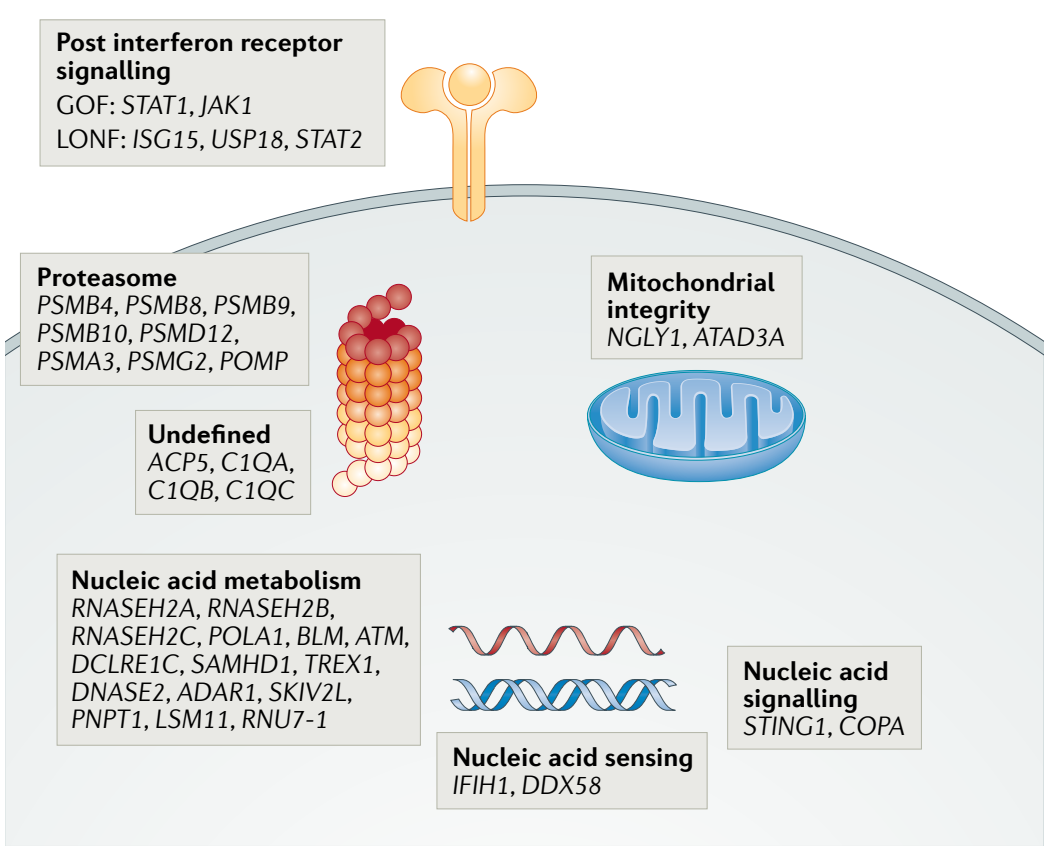

Fig. 2 | Categorization of putative type I interferonopathy genotypes according to the function of the encoded gene product. Seventeen of the 38 putative type I interferonopathy mutated gene products act directly on nucleic acid substrates (nucleic acid 'metabolism') or are involved in nucleic acid sensing, two have a primary role in transducing a DNA signal to an innate immune response, five are involved in downstream regulation of type I interferon receptor signalling and two contribute to the maintenance of mitochondrial integrity. Eight further genes relate to the proteasome, where the precise link to type I interferon induction remains unclear. The relationship of mutations in $A C P 5$ and $C 1 Q A, C 1 Q B$, and $C 1 Q C$ mutations to disease remains unproven to our mind. GOF, gain of function; LONF, loss of negative feedback.

\section{Alu elements}

Part of a family of short

interspersed nuclear elements

that replicate via long

interspersed nuclear

element-mediated reverse

transcription of an RNA

polymerase III transcript. Each Alu element is roughly $280 \mathrm{bp}$

long, followed by a poly(A) tail of variable length. Alu elements are the most abundant sequence repeats in the human genome, making up 5-10\% of the total. recognition of phenotypes associated with enhanced type I interferon signalling becomes more important with the introduction of anti-interferon treatments. In this regard, intriguing recent data have suggested that AGS might be associated with a disturbance of very long chain fatty acid metabolism. Specifically, Armangue et al. ${ }^{41}$ and Tise et al. ${ }^{42}$ described cases of AGS identified through newborn screening programmes for X-linked adrenoleukodystrophy, raising the possibility that AGS might be ascertained presymptomatically. The mechanism here remains unknown, perhaps relating to a disturbance of central nervous system (CNS) myelination or of a link between antiviral signalling and peroxisome integrity worthy of further investigation ${ }^{43,44}$. Alternative biomarkers will be desirable to assess new therapies, and might include indicators of end organ damage not specifically related to underlying pathogenesis (for example, neurofilament light and glial fibrillary acidic protein in the case of the CNS).

\section{Mechanisms of interferon induction}

We can reasonably consider endogenous nucleic acid species driving type I interferon to be of either nuclear or mitochondrial origin. Here we highlight recent insights derived from the study of the type I interferonopathies relating to this point, as well as genotypes associated with a disturbance of DNA stimulus signal transduction, downstream interferon receptor signalling and proteasomal function.
The nucleus. As previously discussed ${ }^{27}$, immunostimulatory nucleic acids originating in the nucleus most likely arise from either chronic DNA damage or the misprocessing of retroelements. While not formally addressed here, the observation of enhanced type I interferon signalling in the context of Bloom syndrome ${ }^{45}$, Artemis deficiency ${ }^{46}$ and ataxia telangiectasia ${ }^{46,47}$ highlights the immunostimulatory potential of products of DNA damage in triggering innate immune signalling. In regard to AGS, the precise nature of the putative stimulating nucleic acid ligands remains uncertain, with the case perhaps most convincingly made for a role of Alu elements related to mutations in $A D A R$, which encodes ADAR1 (REF. ${ }^{48}$.

Cyclic GMP-AMP (cGAMP) synthase (cGAS) is the principal intracellular DNA sensor, catalysing the synthesis of the $2^{\prime}, 3^{\prime}$-linked dinucleotide cGAMP upon DNA binding ${ }^{49,50}$. cGAMP subsequently activates the transmembrane adaptor protein STING (stimulator of interferon genes) on the endoplasmic reticulum (ER), thereby linking DNA detection to TANK-binding kinase 1 (TBK1)-interferon regulatory factor 3 (IRF3) activation. It was previously proposed that the sensing of foreign DNA occurs in the cytosol ${ }^{51}$. However, recent data have placed cGAS in the nucleus ${ }^{52}$, begging the question as to how activation of cGAS in response to host genomic DNA is avoided. Related to this point, in 2020, biallelic mutations in LSM11 and RNU7-1, which are genes encoding components of the replication-dependent histone pre-mRNA processing complex, were described as two additional AGS-associated genotypes ${ }^{53}$. These mutations result in the misprocessing of core and linker histone mRNA transcripts and a disturbance of linker histone protein stoichiometry, leading to an altered distribution of nuclear cGAS, and enhanced interferon signalling mediated by the cGAS-STING pathway. It has been shown that cGAS can bind to, and be activated by, chromatin ${ }^{54}$, and that nucleosomes limit the activation of cGAS by naked DNA ${ }^{55}$. Furthermore, recent cryo-electron microscopy structures have demonstrated that cGAS is physically sequestered away from nuclear DNA through its binding to the acidic patch of the core histones $\mathrm{H} 2 \mathrm{~A}$ and $\mathrm{H}_{2} \mathrm{~B}^{56-60}$. While a disturbance of core histones cannot be ruled out, mutations in LSM11 and RNU7-1 were shown to particularly affect the level of the linker histone H1.4, and the in vitro production of cGAMP by recombinant cGAS was increased following exposure to chromatin lacking linker histone compared with chromatin incorporating it. These data raise the possibility that other histone-related proteins might also play essential physiological roles in limiting cGAS activation within the nuclear compartment.

The mitochondria. Each cell can contain thousands of mitochondria, and nucleic acids with immunostimulatory potential are generated during the transcription and replication of mitochondrial DNA. Mutations in PNPT1 represent the first described Mendelian mitochondrial disease associated with enhanced type I interferon signalling ${ }^{61}$. Bidirectional transcription of the mitochondrial circular genome necessitates the decay of one strand so as to prevent the formation of double-stranded RNA, 
with this function mediated by the mitochondrial degradosome, a complex including the ribonuclease polynucleotide phosphorylase, encoded by PNPT1. Notably, loss of polynucleotide phosphorylase activity resulted in the escape of mitochondrial double-stranded RNA into the cytoplasm and enhanced expression of ISGs through MDA5 in the blood of patients with biallelic PNPT1 mutations. Loss of function of NGLY1, a conserved deglycosylation enzyme, has also been reported to result in chronic activation of cytosolic nucleic acid sensing, this time as a result of mitochondrial fragmentation and the release of immunostimulatory nucleic acid into the cytoplasm ${ }^{62}$, with increased ISG expression observed in patient-derived lymphoblastoid cell lines. More recently, pathogenic mutations in ATAD3A, which encodes the ubiquitously expressed ATPase ATAD3A, have been shown to cause mitochondrial DNAdependent upregulation of type I interferon signalling in the context of autoimmunity (specifically, features of systemic sclerosis) and neurological disease ${ }^{38}$. Ex vivo, there was a marked and persistent elevation of the expression of ISGs and IFNa in the blood, CSF and primary fibroblasts of patients with mutations in ATAD3A. Although the precise mechanism remains unclear, as in the case of NGLY1, these findings possibly result from a leakage

\section{Box 1 | Interferon signalling assays}

\section{Cytopathic protection assay}

Assessment of the ability of patient serum or cerebrospinal fluid (CSF) to protect cells against virus-induced death. The direct link to antiviral activity reflects physiological relevance, but the test is less sensitive in defining mutant status than interferon-stimulated gene (ISG) expression.

\section{Interferon signature}

Quantification of the expression of (a variable number of) ISGs initially chosen on the basis of genome-wide microarray and RNA sequencing. The PAXgene system, with samples travelling stably at room temperature for at least 72 hours, is highly practicable. ISG expression is not specific to any interferon subtype. ISGs cannot normally be assessed in CSF because of low cell numbers.

\section{Single-molecule array}

Measurement of interferon- $\alpha$ (IFN $\alpha$ ) by digital enzyme-linked immunosorbent assay in multiple tissues, including CSF. Costs of the platform/reagents, antibody specificity and the need to transfer material on dry ice are important considerations.

\section{Comments}

- Depending on the clinical context, serial testing may be necessary to minimize the risk of misinterpretation of a 'false positive' result (for example, due to infection).

- The ability to record a disease signal in blood and CSF has proven highly useful, even if neither necessarily represents the relevant tissue in terms of underlying disease (so a negative result does not preclude a role for interferon in pathogenesis).

- Although reliable in many genotypes (for example, the interferon signature is positive in close to $100 \%$ of patients with STING1 mutations), there are exceptions; most particularly, around $25 \%$ of patients with RNASEH2B mutations did not show ISG upregulation when tested after the age of 4 years.

- These tests are not disease specific, being common to a number of apparently distinct phenotypes, such as Aicardi-Goutières syndrome, systemic lupus erythematosus and dermatomyositis.

- There is poor correlation between the interferon score and clinical status, exemplified by individuals with a completely normal phenotype demonstrating marked, and apparently lifelong, upregulation of interferon signalling.

- While the interferon score is an excellent disease biomarker, our experience is it has not behaved as a (highly) reactive biomarker relating to JAK1 inhibition in patients with Aicardi-Goutières syndrome and STING1 mutations, at least at the doses used. of mitochondrial DNA into the cytoplasm, and beg questions as to the ways in which nucleic acids are retained in, and might escape from, mitochondria ${ }^{63}$. The observation that both mitochondrial RNA and mitochondrial DNA can induce interferon signalling raises the possibility that innate immune system engagement may contribute to the phenotype of monogenic mitochondrion-related disorders more generally. Of note, type I interferon induction by mitochondrion-derived nucleic acid has also been implicated in a number of common phenotypes, including Parkinson disease ${ }^{64}$, motor neuron disease $^{65}$ and SLE ${ }^{66}$.

The ER-Golgi apparatus axis. Heterozygous dominantnegative mutations in COPA cause inflammatory arthritis and severe interstitial pulmonary disease ${ }^{35}$. This phenotype overlaps with that seen in patients with STING-associated vasculopathy with onset in infancy $(\mathrm{SAVI})^{67,68}$, where gain-of-function heterozygous STING1 mutations cause constitutive translocation of STING from the ER to the ER-Golgi intermediate compartment ${ }^{69,70}$, a process central to the activation and subsequent termination of type I interferon production in response to cytoplasmic DNA signalling ${ }^{71}$. COPA is a subunit of the coatomer COPI, a complex involved in the retrograde transport of vesicles from the Golgi apparatus to the ER, and recently published results implicate this function as essential in maintaining immunological homeostasis ${ }^{72-74}$. Specifically, these new data suggest that basal cGAS-cGAMP-mediated STING stimulation triggers STING trafficking towards the Golgi apparatus, but that type I interferon pathway activation is limited by the active reverse shuttling of STING back to the $\mathrm{ER}^{75}$. Such retrograde trafficking is mediated by the COPI machinery and involves the sorting protein COPA, which, through the adaptor molecule SURF4, tethers STING as cargo in COPI vesicles. Disruption of Golgi apparatus to ER trafficking, due to dominant-negative mutations in COPA, results in the trapping of STING in the Golgi apparatus and an immune response equivalent to that observed in SAVI. All told, these data highlight the importance of the ER-Golgi apparatus axis in the regulation of STING activation, the potential for manipulating this axis both to dampen and to enhance interferon signalling, and the possibility that other mutant genotypes involved in this pathway might be novel type I interferonopathies.

Interferon receptor signalling. The binding of type I interferons to the receptor subunits interferon- $\alpha / \beta$ receptor 1 (IFNAR1) and IFNAR2 induces the activation of the Janus family tyrosine kinases TYK2 and JAK1. Activated TYK2 and JAK1 in turn phosphorylate STAT1 and STAT2, resulting in formation of the DNA-binding STAT1-STAT2-IRF9 ternary complex ISGF3, which then activates the transcription of genes with an interferon-stimulated response element in their promoters. USP18 exerts a negative regulatory effect on type I interferon signalling by competing with JAK1 for IFNAR2 binding, and mutations in USP18 and ISG15, which directly regulates USP 18 stability, result in aberrant type I interferon induction in humans ${ }^{76,77}$. 
More recently, a third autosomal recessive genotype associated with defective negative regulation of IFNAR signalling was reported ${ }^{78,79}$. Here, a homozygous p.Arg148Trp or p.Arg148Gln separation-of-function mutation in STAT2 results in a failure of the protein to interact with USP18, which is apparently essential for the recruitment of USP18 to IFNAR2 (REF $\left.{ }^{80}\right)$. These three disorders are of particular significance as they implicate a 'pure' defect of unrestrained interferon signalling alone as the cause of disease rather than a specific nucleic acid-sensing pathway. The phenotypic overlap with other type I interferonopathies is notable, particularly with regard to neurological involvement - so much so that we suggest that mutations in USP18 and STAT2 might reasonably be considered as 'severe AGS'. These mutant genotypes provide compelling support for the type I interferonopathy hypothesis, strengthening the clinical rationale for the therapeutic blockade of interferon signalling. Indeed, a child with a homozygous donor splice-site mutation in USP18 demonstrated an apparently favourable response to JAK1 inhibition ${ }^{81}$.

Contrasting mechanistically with loss of postinterferon receptor negative feedback, increased ISG expression in whole blood ${ }^{82}$ and hyperphosphorylation of STAT1 in response to type I interferon in peripheral blood mononuclear cells ${ }^{83}$ is seen with STAT1 gain-of-function mutations. While intracranial calcification $^{84}$ and aortic calcification ${ }^{85}$ have been reported in this context, the core phenotype includes chronic mucocutaneous candidiasis and autoimmune

\section{Box 2 |Phenotypes}

The range of phenotypes encompassed by the type I interferonopathies is remarkably broad. However, a number of clinical entities are easily recognizable, including 'classical' Aicardi-Goutières syndrome (AGS), STING-associated vasculopathy of infancy (SAVI), COPA syndrome and spondyloenchondrodysplasia.

Shared clinical features are seen across the type I interferonopathies, most particularly intracranial calcification and vasculitic skin lesions. Neurological disease with lupus-like stigmata should act as a diagnostic prompt. Interstitial lung (fibrosis/haemorrhage) plus joint disease is characteristic of SAVI and COPA syndrome.

Overviews of clinical phenotype based on important cohorts are available for a number of genotypes, including AGS overall ${ }^{137}$, specific AGS-related genotypes due to mutations in double-stranded RNA-specific adenosine deaminase 1 (ADAR1) (13 $^{13}$ and MDA5 ( $\left(\mathrm{REF}^{31}\right)$, spondyloenchondrodysplasia ${ }^{139}, \mathrm{SAV}^{91}$ and COPA syndrome ${ }^{72,140}$.

Marked phenotypic differences can occur within the same genotype (and the same family). For example, gain-of-function MDA5 mutations can be associated with AGS, non-syndromic spastic paraparesis, Singleton-Merten syndrome and complete clinical non-penetrance into old age. Of passing note, patient 2 in the original description by Singleton and Merten developed a fever and lost the ability to walk aged 14 months in keeping with an AGS-like phenotype.

Clinical non-penetrance is common in interferonopathies inherited as autosomal dominant traits ( $13.5 \%$ for MDA5; perhaps $30 \%$ for COPA syndrome). The rate of non-penetrance in the autosomal recessive disorders is unknown, as clinically asymptomatic siblings are not normally genotyped. While clinically non-penetrant individuals with IFIH1 mutations usually demonstrate robust interferon signalling upregulation, tests of interferon signalling status are close to normal in asymptomatic individuals heterozygous for COPA mutations.

Environmental triggers (infections and vaccination) and additive/protective genetic factors might influence disease onset, expression and flares/progression.

'Outlier' disorders - that is, those showing little clinical overlap with other type I interferonopathies (for example, Bloom syndrome) - likely reflect the importance of distinct functions of the relevant protein and a lesser, if any, contribution of enhanced interferon signalling to the phenotype. hypothyroidism. Gain of function downstream of IFNAR has also been recorded due to JAK1 mutations, which are associated with a complex multisystem inflammatory phenotype ${ }^{86,87}$. It is important to note that both of these disorders involve the triggering of multiple cytokine pathways in addition to type I interferon signalling, likely explaining aspects of their phenotypes not observed in other type I interferonopathies.

Proteasome. Loss of function of proteasome-related proteins appears to be consistently associated with enhanced type I interferon signalling, with the number of genes so far identified - eight in total - outnumbered only by the bewildering array of alternative names and acronyms coined. Here, as first suggested by Sanchez et al. ${ }^{88}$, we refer to this group of disorders as 'proteasome-associated autoinflammatory syndromes' (PRAAS), variably encompassing early-onset neutrophilic infiltrative skin disease, panniculitis and lipodystrophy, blood dyscrasias (anaemia and thrombocytopenia), immunological dysfunction (for example, lymphopenia, dysgammaglobulinaemia and immunodeficiency), muscle atrophy, joint contractures and intracranial calcification. Proteasomes are heteromultimeric protein complexes found in all eukaryotic cells that catalyse the non-lysosomal proteolytic degradation of ubiquitinylated proteins ${ }^{89}$. Known PRAAS-associated mutations occur in genes encoding constitutive proteasome subunits (PSMB4 and PSMA3), immunoproteasome subunits (PSMB8, PSMB9 and PSMB10), the $19 S$ regulatory particle (PSMD12) and proteasome assembly factors (POMP and PSMG2). Brehm et al. ${ }^{90}$ made the notable observation of 'additive' loss-offunction mutations in proteasome components conforming to a pattern of digenic inheritance. Mutations in these genes result in decreased proteasome activity, an aberrant accumulation of cytosolic ubiquitinylated protein conjugates and activation of an unfolded protein response. Importantly, these mutant genotypes are also associated with a marked induction of ISG expression. While several groups are known to be working in this area, the mechanism by which decreased proteasome activity causes innate immune activation awaits elucidation (with Brehm et al. ${ }^{90}$ indicating that the pathway involved was not dependent on STING).

\section{Phenotypes, expression and penetrance}

Given the hypothesized fundamental role of type I interferon in the pathogenesis of type I interferonopathies, we hold that the type I interferonopathies should be considered as autoinflammatory in basis, with 'spillover' into autoimmunity in some cases. Discrete phenotypic labels can be important for affected families and individuals when thinking about prognosis (BOX 2; TABLE 1). At the same, the premise of the type I interferonopathy categorization is that these disorders have a common pathology (and, possibly, route to therapy), so we think that there is value in the use of the umbrella term type I interferonopathies. There is a striking overlap of clinical features, particularly the involvement of the brain and skin, across several type interferonopathies. However, clear phenotypic differences also exist between 
Table 1 | Genotypes linked to putative type I interferonopathies

\begin{tabular}{|c|c|c|c|c|}
\hline Gene & Protein function & $\begin{array}{l}\text { Proposed link to type I } \\
\text { interferon signalling }\end{array}$ & Mutation effect & Phenotypic label/features \\
\hline TREX1 & Deoxyribonuclease & Cytosolic DNA & $\begin{array}{l}\text { LOF (autosomal recessive } \\
\text { or dominant negative) }\end{array}$ & AGS, FCL, SLE \\
\hline DNASE2 & Deoxyribonuclease & Cytosolic DNA & LOF (autosomal recessive) & $\begin{array}{l}\text { Neonatal anaemia, glomerulonephritis, } \\
\text { liver fibrosis, deforming arthropathy }\end{array}$ \\
\hline SAMHD1 & Control of dNTP pool & Cytosolic DNA & LOF (autosomal recessive) & AGS, FCL, cerebrovascular disease \\
\hline STING1 & $\begin{array}{l}\text { Cytosolic DNA signal } \\
\text { transduction }\end{array}$ & Cytosolic DNA & GOF (autosomal dominant) & $\begin{array}{l}\text { STING-associated vasculopathy with } \\
\text { onset in infancy, FCL }\end{array}$ \\
\hline RNASEH2A & Ribonuclease & Cytosolic RNA-DNA hybrids & LOF (autosomal recessive) & AGS \\
\hline RNASEH2B & Ribonuclease & Cytosolic RNA-DNA hybrids & LOF (autosomal recessive) & AGS, spastic paraparesis \\
\hline RNASEH2C & Ribonuclease & Cytosolic RNA-DNA hybrids & LOF (autosomal recessive) & AGS \\
\hline POLA1 & DNA polymerase & Cytosolic RNA-DNA hybrids & LOF (X-linked recessive) & $\mathrm{X}$-linked reticulate pigmentary disorder \\
\hline ADAR1 & RNA editing & Cytosolic dsRNA & $\begin{array}{l}\text { LOF (autosomal recessive } \\
\text { or dominant negative) }\end{array}$ & $\begin{array}{l}\text { AGS, dyschromatosis symmetrica } \\
\text { hereditaria, bilateral striatal necrosis, } \\
\text { spastic paraparesis }\end{array}$ \\
\hline IFIH1 & dsRNA sensor & Cytosolic dsRNA & GOF (autosomal dominant) & $\begin{array}{l}\text { AGS, spastic paraparesis, Singleton- } \\
\text { Merten syndrome }\end{array}$ \\
\hline DDX58 & dsRNA sensor & Cytosolic dsRNA & GOF (autosomal dominant) & $\begin{array}{l}\text { Singleton-Merten syndrome, juvenile } \\
\text { open-angle glaucoma }\end{array}$ \\
\hline SKIV2L & RNA helicase & Cytosolic RNA (UPR) & LOF (autosomal recessive) & Trichohepatoenteric syndrome \\
\hline LSM11 & RDH pre-mRNA processing & $\begin{array}{l}\text { Histone stoichiometry/ } \\
\text { genomic DNA }\end{array}$ & LOF (autosomal recessive) & AGS \\
\hline RNU7-1 & RDH pre-mRNA processing & $\begin{array}{l}\text { Histone stoichiometry/ } \\
\text { genomic DNA }\end{array}$ & LOF (autosomal recessive) & AGS \\
\hline PNPT1 & Polynucleotide phosphorylase & Mitochondrial RNA & LOF (autosomal recessive) & $\begin{array}{l}\text { Infantile encephalopathy, bilateral } \\
\text { striatal necrosis }\end{array}$ \\
\hline NGLY1 & N-deglycosylation & $\begin{array}{l}\text { Mitochondrial DNA and } \\
\text { RNA (indirect) }\end{array}$ & LOF (autosomal recessive) & $\begin{array}{l}\text { Infantile encephalopathy, movement } \\
\text { disorder }\end{array}$ \\
\hline ATAD3A & Multiple & Mitochondrial DNA & $\begin{array}{l}\text { Dominant negative } \\
\text { (autosomal dominant) }\end{array}$ & $\begin{array}{l}\text { Global developmental delay, systemic } \\
\text { sclerosis, spastic paraparesis }\end{array}$ \\
\hline ATM & dsDNA break repair & dsDNA breaks & LOF (autosomal recessive) & Ataxia telangiectasia \\
\hline DCLRE1C & dsDNA break repair & dsDNA breaks & LOF (autosomal recessive) & Immunodeficiency \\
\hline$B L M$ & Genome stability & DNA damage & LOF (autosomal recessive) & Bloom syndrome \\
\hline COPA & $\begin{array}{l}\text { Vesicle transport (ER to Golgi } \\
\text { apparatus) }\end{array}$ & STING trafficking & $\begin{array}{l}\text { Dominant negative } \\
\text { (autosomal dominant) }\end{array}$ & $\begin{array}{l}\text { Interstitial lung disease, pulmonary } \\
\text { haemorrhage, arthropathy, } \\
\text { glomerulonephritis }\end{array}$ \\
\hline ISG15 & Inhibition of ISG transcription & IFNAR2 signalling & LOF (autosomal recessive) & $\begin{array}{l}\text { Intracranial calcification, Mendelian } \\
\text { susceptibility to mycobacterial disease }\end{array}$ \\
\hline UPS18 & Inhibition of ISG transcription & IFNAR2 signalling & LOF (autosomal recessive) & AGS-like \\
\hline STAT2 & Inhibition of ISG transcription & IFNAR2 signalling & $\begin{array}{l}\text { LOF (autosomal recessive; } \\
\text { separation-of-function) }\end{array}$ & AGS-like \\
\hline STAT1 & Cytokine signalling & ISG signalling & GOF (autosomal dominant) & $\begin{array}{l}\text { Chronic mucocutaneous candidiasis, } \\
\text { immunodeficiency, autoimmunity, } \\
\text { intracranial calcification }\end{array}$ \\
\hline JAK1 & Cytokine signalling & ISG signalling & GOF (autosomal dominant) & Eosinophilia, atopy \\
\hline C1QA & $\begin{array}{l}\text { Alternative complement } \\
\text { pathway }\end{array}$ & $\begin{array}{l}\text { Immune complexes/ } \\
\mathrm{CD} 8^{+} \mathrm{T} \text { cell metabolism }\end{array}$ & LOF (autosomal recessive) & SLE \\
\hline C1QB & $\begin{array}{l}\text { Alternative complement } \\
\text { pathway }\end{array}$ & $\begin{array}{l}\text { Immune complexes/ } \\
\text { CD8 }{ }^{+} \text {T cell metabolism }\end{array}$ & LOF (autosomal recessive) & SLE \\
\hline C1QC & $\begin{array}{l}\text { Alternative complement } \\
\text { pathway }\end{array}$ & $\begin{array}{l}\text { Immune complexes/ } \\
\mathrm{CD} 8^{+} \mathrm{T} \text { cell metabolism }\end{array}$ & LOF (autosomal recessive) & SLE \\
\hline ACP5 & Phosphatase & $\begin{array}{l}\text { Phosphorylation of } \\
\text { osteopontin }\end{array}$ & LOF (autosomal recessive) & Spondyloenchondrodysplasia, SLE \\
\hline PSMB8 & Proteasome & Unknown & LOF (autosomal recessive) & PRAAS \\
\hline
\end{tabular}


Table 1 (cont.) | Genotypes linked to putative type I interferonopathies

\begin{tabular}{|lllll} 
Gene & Protein function & $\begin{array}{l}\text { Proposed link to type I } \\
\text { interferon signalling }\end{array}$ & Mutation effect & Phenotypic label/features \\
\hline PSMB4 & Proteasome & Unknown & LOF (autosomal recessive) & PRAAS \\
\hline PSMA3 & Proteasome & Unknown & LOF (autosomal recessive) & PRAAS \\
\hline PSMB9 & Proteasome & Unknown & LOF (autosomal recessive) & PRAAS \\
\hline POMP & Proteasome & Unknown & $\begin{array}{l}\text { Dominant negative } \\
\text { (autosomal dominant) }\end{array}$ & PRAAS \\
\hline PSMB10 & Proteasome & Unknown & LOF (autosomal recessive) & PRAAS \\
\hline PSMG2 & Proteasome & Unknown & LOF (autosomal recessive) & PRAAS \\
\hline PSMB12 & Proteasome & Unknown & LOF (autosomal dominant) & Global developmental delay \\
\hline
\end{tabular}

The table provides an overview of genotypes considered as consistently associated with enhanced type I interferon signalling, with protein function, link to interferon signalling, proposed molecular mechanism and categorization of currently recognized associated clinical phenotypes. ADAR1, double-stranded RNA-specific adenosine deaminase 1; AGS, Aicardi-Goutières syndrome; COPA, coatomer subunit- $\alpha$; dNTP, deoxynucleoside triphosphate; dsDNA, double-stranded DNA; dsRNA, double-stranded RNA; ER, endoplasmic reticulum; FCL, familial chilblain lupus; GOF, gain of function; ISG, interferon stimulated gene; LOF, loss of function; PRAAS, proteasome-associated autoinflammatory syndrome; RDH, replication-dependent histone; SLE, systemic lupus erythematosus; UPR, unfolded protein response.

some of these genotypes; for example, the severe lung disease seen with mutations in STING1 and COPA is not a feature previously noted in the context of other putative type I interferonopathies. Furthermore, apparently distinct complex diseases, particularly SLE and dermatomyositis, are also characterized by enhanced type I interferon signalling. The basis of such variable expression remains unclear; indeed, the diversity of clinical phenotypes associated with a type I interferon signature might be considered as undermining the type I interferon hypothesis per se (together with the observation of clinical non-penetrance despite enhanced type I interferon signalling - see later). Distinct expression patterns of disease-associated proteins, their protein partners, interferon-inducing signalling components and proteins involved in alternative ('redundant') signalling pathways might all be relevant here. Some preliminary light has been shed on this topic recently with the simple observation that while IFNa is present in the CSF and serum of a number of interferon-related diseases, the primary sites of interferon production in AGS (a neurologically focused phenotype) and SAVI (where neurological disease is rare) are, respectively, the CNS and the periphery ${ }^{30}$. Whereas STING signalling is central to seven of nine AGS-associated genotypes and all SAVI cases (FIG. 1), an important distinction is that endogenous nucleic acid ligands activate cGAS-cGAMP-STING signalling in AGS, but in SAVI mutant STING proteins are constitutively active without a requirement for ligand engagement.

Clinical non-penetrance (or age-related penetrance in some cases) is a common feature of the dominant negative p.Gly1007Arg mutation in ADAR1, the known dominant negative mutations in TREX1, and heterozygous mutations in COPA and IFIH1 (dominant negative and gain of function, respectively). In a recent study of 74 individuals harbouring pathogenic lesions in IFIH1, $13.5 \%$ of these individuals (seven of whom were older than 50 years) were clinically asymptomatic ${ }^{31}$. For those with dominant negative mutations in COPA, the rate of clinical non-penetrance is estimated from the literature at around $30 \%$. By contrast, reported carriers of a STING gain-of-function mutation are almost invariably symptomatic, although phenotypic variability (variable expression) can be observed (even within the same family $)^{91}$. Of note, while clinical non-penetrance is a feature of MDA5 gain of function, such asymptomatic individuals usually demonstrate a persistent upregulation of ISG transcripts ${ }^{31}$, contrasting with COPA syndrome, where the interferon signature is apparently normal or only minimally elevated in asymptomatic carriers $^{72}$. The significance of this observation is unclear.

We draw attention to the fact that while variable expression is well recognized in AGS inherited in an autosomal recessive manner, the rate of clinical nonpenetrance in such cases is unknown - because asymptomatic siblings are not typically genotyped. In this regard, we note the report of a woman first developing cutaneous features at the age of 19 years and demonstrating no abnormal neurological features at the age of 32 years in the context of a biallelic p.Ala177Thr mutation in RNase $\mathrm{H} 2 \mathrm{~b}^{92}$. Broadly speaking, given intrafamilial and interfamilial discrepancies related to the same mutation, such differences in penetrance (and expression) must relate to other factors, genetic or environmental. Although mosaicism might explain occasional cases $^{93,94}$, non-penetrance is well described in germline inheritance. The possibility of a 'cumulative' genetic burden is illustrated by the data from the study of PRAAS mentioned earlier herein ${ }^{90}$. Infection has been noted as a potential trigger of disease in ADAR1-related bilateral striatal necrosis ${ }^{95}$; whether vaccination might act similarly is currently unclear. In this regard, it is important to note that most of the type I interferonopathy-related genes, as well as the key components of the DNA-sensing and RNA-sensing pathways that they regulate, are strongly inducible by interferons. Thus, one can envision a simple gene-environment relationship in which infection could induce the expression of key sensors above a critical threshold, which then causes aberrant detection of self nucleic acids and progression to symptomatic disease $^{96}$. Age at exposure to a given environmental factor might also be important, perhaps reflecting changes in the immune system over time (akin to the situation with the risk of invasive pneumococcal disease in deficiency of IRAK4 and MYD88 (REF. $\left.{ }^{97}\right)$ ). The question 
remains as to whether clinically asymptomatic individuals are at risk of developing later-onset disease. An excellent overview of the general principles underlying penetrance in immunological disease is available ${ }^{98}$.

\section{Mouse models of the type I interferonopathies}

Our understanding of the biology of the type I interferonopathies has been informed by a number of mouse models defining the relationships between Mendelian interferonopathy gene products and the innate immune pathways that they regulate. Thus, it is now well established that TREX1, RNase $\mathrm{H} 2$ and SAMHD1 are specific regulators of the cGAS-STING DNA-sensing pathway ${ }^{99-104}$, whereas the RNA-editing enzyme ADAR1 prevents MDA5-MAVS activation by self RNAs ${ }^{105-107}$. Additionally, mouse data indicate that SAVI-associated mutations activate STING signalling without a requirement for $\mathrm{cGAS}^{108}$. These elegantly simple relationships are particularly appealing examples of how self versus non-self discrimination is achieved by the innate immune system, and have motivated billions of dollars of investment in the development of pathway-specific inhibitors that hold new promise for the treatment of AGS and other interferonopathies. Importantly, these observations provide a road map for patient stratification that is essential for the deployment of any new therapy. For example, cGAS inhibitors would be predicted as useful in AGS (excluding AGS due to mutations in ADAR and IFIH1) but not in SAVI, whereas STING inhibition might have therapeutic value in both of these settings. A fascinating and fundamental question is whether the putative immunostimulatory endogenous ligands

\section{Box 3 | Treatments for type I interferonopathies}

The type I interferonopathy concept implies shared pathology, and the schema in FIG. 2 suggests common approaches to therapy: including limiting the production or enhancing the removal of putative self nucleic acid stimuli and blocking signalling downstream of such stimuli.

\section{Points for consideration in treating distinct type I interferonopathies}

- The relative contribution of interferon-mediated and interferon-independent aspects of a clinical phenotype needs to be kept in mind.

- What is the window of therapeutic opportunity - that is, at what stage in the disease process must treatment start to avoid irreversible tissue damage?

- Is the target disease a progressive or a static disorder? Thus, is treatment useful only early in the disease process, or is there utility in lifelong treatment, or treatment in association with disease flares?

- Variable disease expression, including complete non-penetrance, can make the assessment of therapeutic efficacy difficult on clinical grounds alone, emphasizing the importance of reactive biomarkers.

- Tissue accessibility, such as central nervous system penetration, is important.

- Is there a role for haematopoietic stem cell or discrete organ (for example, the lungs in STING-associated vasculopathy of infancy and COPA syndrome) transplant in any of the type I interferonopathies?

- Randomization and blinded studies can be challenging in rare disorders with early onset and high mortality.

-Where the aim is to block interferon signalling, the risk of infectious susceptibility needs to be taken into account.

- Combinatorial therapy may be beneficial.

- Experimental medicine approaches are likely to be the only way to assess the true value of different therapeutic strategies in humans. underlying the type I interferonopathies are relevant to, and can be used to stratify, complex interferon-associated diseases such as SLE, for which the precise genetic contributions remain obscure ${ }^{96,109,110}$.

Despite the importance of these models as experimentally tractable systems to define disease mechanisms, they remain an imperfect approximation of the disease phenotypes that are observed in the corresponding human disease states. Of particular note, the neurological involvement so characteristic of AGS has not been convincingly recorded in any murine system. For example, TREX1-deficient mice develop severe inflammatory myocarditis ${ }^{11}$ in the absence of CNS disease. Moreover, Samhd $1^{-/}$mice present with a mild interferon signature and no tissue disease at all ${ }^{104}$, in contrast to AGS due to SAMHD1 loss of function. Finally, Adar-mutant mice, which model the most common mutation (p.Pro193Ala) in ADAR1-related AGS, demonstrate kidney and liver dysfunction but no evidence of brain involvement ${ }^{112}$. Why are these mouse phenotypes so different from the human diseases that they model? We propose two potential explanations. First, the cell types and tissues that express the relevant sensors and essential negative regulators might not overlap perfectly between humans and mice. Second, the source, abundance and tissue distribution of the endogenous nucleic acids that are acted upon by AGS-related enzymes may differ between humans and mice, with the endogenous RNAs that are ADAR1 substrates and MDA5 ligands a particularly illustrative example. Here, recent evidence implicates inverted repeats of Alu elements in non-coding regions of mRNAs as an abundant reservoir of double-stranded RNAs that are edited by ADAR1 and can potently activate MDA5 in ADAR-mutant human cells ${ }^{48,113}$. However, Alu elements do not exist in the mouse genome. Instead, mice harbour millions of copies of B2 short interspersed nuclear elements, which, like Alu elements, are derived from 7SL non-coding RNA. B2 short interspersed nuclear elements are half the length of Alu elements, and their distribution in the mouse genome does not overlap with that of Alu elements in the human genome. Therefore, the most compelling source of endogenous RNAs relevant for $A D A R$ mutation-driven AGS in humans does not exist in mice. A better understanding of the tissue-specific and species-specific triggers of the type I interferonopathies will provide a framework for exploring the biochemistry and cell biology of nucleic acid metabolism and how it relates to other immune diseases.

\section{Treatments}

While a set of mutant genotypes associated with an upregulation of interferon signalling clearly exists, a more important question, at least clinically, is the relevance of this interferon upregulation to phenotype, and thus the potential efficacy of anti-interferon therapy (BOX 3). The convergence of most of the type I interferonopathy genotypes on nucleic acid metabolism and sensing, data from mouse models demonstrating a phenotypic dependence on type I interferon receptor signalling ${ }^{17,112,114}$, overlap of clinical features (for example, intracranial calcification seen in more than half of 
mutant genotypes; as well as the induction of stigmata of the type I interferonopathies in the context of treatment with type I interferon, (for example, REF. ${ }^{115}$ )) and the diseases related to discrete negative regulation of the type I interferon receptor discussed earlier herein all support a primary role of enhanced interferon signalling in driving disease (BOX 4). On the other hand, certain mouse data $^{116,117}$, imperfect correlations between interferon biomarker status and disease penetrance, and the induction of other proinflammatory cytokines beyond the interferon transcriptional programme (for example, REF. ${ }^{118}$ ) suggest that interferon signalling might not be central to disease in all cases. In this regard, three groups have recently shown robust STING-dependent antiviral and antitumour activity that is independent of interferon, possibly mediated by NF- $\kappa B^{119-121}$. Of course, interferon signalling might contribute to disease, but not necessarily exclusively so, and disease due to DNASE2 mutations is possibly instructive here. Thus, DNASE2-null mice accumulate undigested DNA in the lysosomes of macrophages, which then chronically activates type I interferon production and results in a lethal perinatal anaemia $^{122}$. Deletion of the type I interferon receptor gene rescues Dnase $2 a$-knockout mice from this lethal anaemia ${ }^{123}$, but these double-knockout mice subsequently develop chronic polyarthritis ${ }^{124}$. Both of these embryonic and postnatal phenotypes are cGAS and STING dependent, indicating dual interferon-mediated and non-interferon-mediated pathways that can drive disease downstream of STING. Therefore, the efficacy of interferon blockade therapy might depend on the 'purity' of an interferonopathy phenotype; that is, how much of the phenotype is due to interferon upregulation and how much is related to other functions of the protein (for example, the role of BLM in DNA

\section{Box 4 | Does interferon drive disease in the putative type I interferonopathies?}

\section{Supporting evidence}

- Correlation of enhanced type I interferon signalling with mutant status.

- Clustering of mutant genotypes with a known role in nucleic acid processing, type I interferon signalling, and in negative feedback of interferon-stimulated gene expression.

- Phenotypic resemblance to in utero-acquired infection in certain cases.

- Phenotypic overlap across genotypes.

- Recapitulation of the neuropathology of Aicardi-Goutières syndrome in a transgenic mouse model chronically producing interferon- $\alpha$ (IFNa) from astrocytes.

- The side effect profile of IFNa used therapeutically.

- Phenotypic rescue in certain murine models when crossed with type I interferon receptor-null mice.

- Apparent clinical efficacy of JAK1 inhibitors (premised on, but not proven to act through, the blocking of type I interferon receptor signalling).

\section{Contradictory evidence}

- Clinical non-penetrance despite enhanced type I interferon signalling.

- The absence of an interferon signature (at least in blood) in some individuals with a mutant genotype.

- The diversity of clinical phenotype associated with a type I interferon signature.

- The observation of phenotypes in STING gain-of-function mice that are not dependent on type I interferon.

- Knowledge that non-interferon inflammatory molecules can also be induced by nucleic acid signalling, in particular NF- $\mathrm{kB}$ through STING. repair, or the consequence of dysfunction of ATAD3A for oxidative phosphorylation) and/or induction of non-interferon signalling pathways (through, for example, NF-kB) (FIG. 3).

In general terms, targeted treatments in the type I interferonopathies might aim at limiting the production or enhancing the removal of putative self nucleic acid stimuli and/or blocking signalling downstream of such stimuli. Related to this latter point, a recently developed mouse model of ADAR1 dysfunction was rescued both by crossing it with an Ifnar 1-knockout mouse and also by treatment with an inhibitor of the integrated stress response (triggered downstream of the eIF2 $\alpha$ kinase PKR) ${ }^{112}$. However, considering specific treatment approaches already trialled in humans, two are of note. Firstly, premised on a reduction of immunostimulatory DNA derived through a reverse transcription (RNA > DNA) step in the life cycle of endogenous retroelements ${ }^{17,125}$, Rice et al. ${ }^{126}$ undertook an open-label pilot study of reverse transcriptase inhibitors in AGS. Patients with mutations in AGS1, AGS2, AGS3, AGS4 or AGS5 (that is, excluding ADAR-mutant and IFIH1-mutant genotypes, where disease is mediated via an RNA signalling pathway) were treated with abacavir, lamivudine and zidovudine for 12 months, and interferon status was assessed before, during and after treatment. The results indicated an effect of treatment in reducing interferon signalling, and possibly increasing cerebral blood flow. A follow-up study is now planned (NCT04731103). While the use of reverse transcriptase inhibitors remains experimental, small case reports (for example, REFS ${ }^{127-129}$ ) and four larger observational studies ${ }^{91,130-132}$ (summarized in REF. ${ }^{133}$ ) have described encouraging results of the inhibition of JAK1 in several type I interferonopathies in a clinical setting. Clear effects on associated systemic and skin disease indicate that these drugs address a relevant biological pathway. However, the effect on neurological features remains more difficult to assess. Notably in this regard, Neven et al. ${ }^{134}$ described the onset of AGS at the age of 15 months in a child with biallelic mutations in RNASEH2B, despite treatment with ruxolitinib (a JAK1 and JAK2 inhibitor) starting at the age of 5 months, when the child was asymptomatic. The study authors observed drug concentrations in the CSF to be only $10 \%$ of those in blood, suggesting the importance of CNS drug penetration. The observation of apparent neurological benefit in a child with biallelic mutations in USP18 following an increase in the dosage of ruxolitinib from 5 to $10 \mathrm{mg}$ twice daily might relate to this same point ${ }^{81}$.

The future of treatments relevant to the type I interferonopathies holds considerable promise, with the rapid development in the biotechnology industry of inhibitors of cGAS and STING. Inhibitors of the RIG-I-like receptors, including MDA5, have not yet been described, but are of similar potential interest. All told, the confluence of human genetics and mechanistic definition of the type I interferonopathies has set the stage for a precision medicine approach in this field, matching the specific source of aberrant innate immune signalling to targeted therapy. 


\section{Conclusions}

The clustering of mutant genotypes implicated in nucleic acid metabolism and sensing and in negative regulation of type I interferon signalling - in some cases, for example, DNASE2 deficiency and ATAD3A-related disease, identified through agnostic screening for type I interferon upregulation - provides compelling evidence in support of the type I interferonopathy hypothesis. Notable recent insights derived from the definition and study of this rare disease grouping include the identification of physiologically important Golgi apparatus to ER transport of STING involving $\mathrm{COPA}^{72-74}$, the implication of innate immune system activation as a contributor to the phenotype of certain monogenic mitochondrial diseases ${ }^{38,61}$, the characterization of a separation-of-function mutation in STAT2 specifically affecting its role in limiting IFNAR2 signalling ${ }^{78,79}$ and the observation that disturbed histone stoichiometry due to mutations in LSM11 and RNU7-1 enhances the immunostimulatory potential of nuclear $\mathrm{DNA}^{53}$. All of these findings inform possible novel drug targets relevant to the future treatment of diseases associated with enhanced type I interferon signalling.

Many important questions central to a proper understanding of the type I interferonopathies remain, perhaps the most interesting being the precise molecular drivers, nucleic acid or otherwise, of aberrant cytokine production. Of particular note, at the time of writing of this Review, the mechanism by which proteasomal dysfunction induces type I interferon signalling is unresolved, and how mutations in lysosomal ACP5 result in such a highly penetrant Mendelian mimic of SLE - namely, spondyloenchondrodysplasia - is also deserving of more detailed study. Furthermore, the precise contribution of non-interferon-mediated signalling to clinical disease is unclear in many of these mutant genotypes, and is of fundamental relevance when one is considering approaches to their treatment - an issue that may be resolved only through clinical experimentation in humans.

The broader implications deriving from the study of the type I interferonopathies remain to be determined. Most obviously, the link to SLE and dermatomyositis, both associated with enhanced type I interferon signalling, is likely a fertile ground for investigation, with a number of articles highlighting a link between these

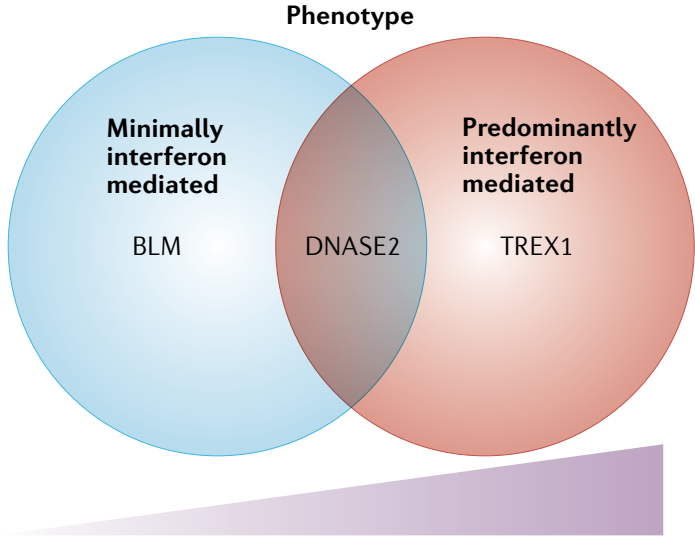

Predicted efficacy of 'anti-interferon' therapy

Fig. 3 | Relationship between the relative contribution of interferon-mediated and non-interferon-mediated inflammation to putative type I interferonopathy phenotypes. The effect of 'anti-interferon' treatments is expected to depend on the contribution of upregulated interferon signalling to the associated phenotype. Exemplar genotypes are given where the clinical efficacy of such therapies is expected to differ. Other factors (for example, the stage in the disease process at which treatment is started) will likely also be important in determining therapeutic efficacy.

monogenic mutant genotypes and 'non-syndromic' systemic lupus erythematosus (for example, REFS ${ }^{109,135,136}$ ). Aligned with this point, it is tempting to speculate that polymorphisms in nucleic acid receptors and associated signalling components might confer a selective advantage in fighting infection ${ }^{96}$, noting, for example, that approximately 1 in 300 and approximately 1 in 440 persons of European (non-Finnish) ancestry are heterozygous for the p.Pro193Ala and p.Ala177Thr mutations in ADAR1 and RNase H2B, respectively.

In summary, the past 10 years have witnessed a major expansion in the number of putative type I interferonopathies, knowledge of their pathogenesis and approaches to their treatment. We look forward to further exciting developments in this field in the near-term.

Published online 20 October 2021
1. Crow, Y. J. Type I interferonopathies: a novel set of inborn errors of immunity. Ann. N. Y. Acad. Sci. 1238 91-98 (2011).

2. Crow, Y. J., Lebon, P., Casanova, J. L. \& Gresser, I. A brief historical perspective on the pathological consequences of excessive Type I interferon exposure in vivo. J. Clin. Immunol. 38, 694-698 (2018).

3. Gresser, I. Can interferon induce disease? Interferon 4 95-127 (1982).

A summary of early data suggesting that type I interferon might be harmful in humans, as first suggested by Gresser.

4. Casanova, J. L. Ion Gresser. J. Interferon Cytokine Res. 39, 317-320 (2019)

5. Lebon, P. et al. Intrathecal synthesis of interferonalpha in infants with progressive familial encephalopathy. J. Neurol. Sci. 84, 201-208 (1988).

6. Aicardi, J. \& Goutieres, F. A progressive familial encephalopathy in infancy with calcifications of the basal ganglia and chronic cerebrospinal fluid lymphocytosis. Ann. Neurol. 15, 49-54 (1984).
7. Smedley, H., Katrak, M., Sikora, K. \& Wheeler, T. Neurological effects of recombinant human interferon. Br. Med. J. 286, 262-264 (1983).

8. Vesikari, T., Nuutila, A. \& Cantell, K. Neurologic sequelae following interferon therapy of juvenile laryngeal papilloma. Acta Paediatr. Scand. 77, 619-622 (1988).

9. Ronnblom, L. E., Alm, G. V. \& Oberg, K. E. Possible induction of systemic lupus erythematosus by interferon-alpha treatment in a patient with a malignant carcinoid tumour. J. Intern. Med. 227 207-210 (1990).

10. Tolaymat, A., Leventhal, B., Sakarcan, A., Kashima, H. $\&$ Monteiro, C. Systemic lupus erythematosus in a child receiving long-term interferon therapy. J. Pediatr 120, 429-432 (1992)

11. Akwa, Y. et al. Transgenic expression of IFN-alpha in the central nervous system of mice protects against lethal neurotropic viral infection but induces inflammation and neurodegeneration. J. Immunol. 161, 5016-5026 (1998)

The first description of a mouse mode demonstrating CNS upregulation of interferon signalling.
12. Campbell, I. L. et al. Structural and functional neuropathology in transgenic mice with CNS expression of IFN-alpha. Brain Res. 835, 46-61 (1999).

13. Crow, Y. J. et al. Cree encephalitis is allelic with Aicardi-Goutieres syndrome: implications for the pathogenesis of disorders of interferon alpha metabolism. J. Med. Genet. 40, 183-187 (2003).

14. Crow, Y. J. et al. Mutations in the gene encoding the 3'-5' DNA exonuclease TREX1 cause Aicardi-Goutieres syndrome at the AGS1 locus. Nat. Genet. 38, 917-920 (2006).

15. Crow, Y. J. et al. Mutations in genes encoding ribonuclease $\mathrm{H} 2$ subunits cause Aicardi-Goutieres syndrome and mimic congenital viral brain infection. Nat. Genet. 38, 910-916 (2006).

16. Rice, G. I. et al. Mutations involved in AicardiGoutieres syndrome implicate SAMHD1 as regulator of the innate immune response. Nat. Genet. 41 829-832 (2009).

17. Stetson, D. B., Ko, J. S., Heidmann, T. \& Medzhitov, R. Trex 1 prevents cell-intrinsic initiation of autoimmunity. Cell 134, 587-598 (2008). 
18. Briggs, T. A. et al. Tartrate-resistant acid phosphatase deficiency causes a bone dysplasia with autoimmunity and a type I interferon expression signature. Nat. Genet. 43, 127-131 (2011)

19. Lausch, E. et al. Genetic deficiency of tartrate-resistant acid phosphatase associated with skeletal dysplasia, cerebral calcifications and autoimmunity. Nat. Genet 43, 132-137 (2011).

20. Lood, C. et al. C1q inhibits immune complex-induced interferon-alpha production in plasmacytoid dendritic cells: a novel link between $\mathrm{C} 1 \mathrm{q}$ deficiency and systemic lupus erythematosus pathogenesis. Arthritis Rheum. 60, 3081-3090 (2009)

21. Santer, D. M. et al. C1q deficiency leads to the defective suppression of IFN-alpha in response to nucleoprotein containing immune complexes. J. Immunol. 185, 4738-4749 (2010).

22. Manthiram, K., Zhou, Q., Aksentijevich, I. \& Kastner, D. L. The monogenic autoinflammatory diseases define new pathways in human innate immunity and inflammation. Nat. Immunol. 18 , 832-842 (2017)

23. Notarangelo, L. D., Bacchetta, R., Casanova, J. L. \& Su, H. C. Human inborn errors of immunity: An expanding universe. Sci. Immunol. 5, eabb1662 (2020).

24. Casanova, J. L. \& Abel, L. Lethal infectious diseases as inborn errors of immunity: toward a synthesis of the germ and genetic theories. Annu. Rev. Pathol. 16, 23-50 (2021)

25. Ablasser, A. \& Hur, S. Regulation of cGAS- and RLRmediated immunity to nucleic acids. Nat. Immunol. 21, 17-29 (2020)

26. Hopfner, K. P. \& Hornung, V. Molecular mechanisms and cellular functions of cGAS-STING signalling. Nat. Rev. Mol. Cell. Biol. 21, 501-521 (2020).

27. Uggenti, C., Lepelley, A. \& Crow, Y. J. Self-Awareness: Nucleic Acid-Driven Inflammation and the Type I Interferonopathies. Annu. Rev. Immunol. 37, 247-267 (2019)

28. Rice, G. I. et al. Assessment of interferon-related biomarkers in Aicardi-Goutieres syndrome associated with mutations in TREX1, RNASEH2A, RNASEH2B, RNASEH2C, SAMHD 1, and ADAR: a case-control study. Lancet Neurol. 12, 1159-1169 (2013).

29. Rodero, M. P. et al. Detection of interferon alpha protein reveals differential levels and cellular sources in disease. J. Exp. Med. 214, 1547-1555 (2017)

30. Lodi, L. et al. Differential expression of interferonalpha protein provides clues to tissue specificity across type I interferonopathies. J. Clin. Immunol. 41 603-609 (2021)

31. Rice, G. I. et al. Genetic and phenotypic spectrum associated with IFIH1 gain-of-function. Hum. Mutat 41, 837-849 (2020)

32. Rice, G. I. et al. Gain-of-function mutations in IFIH cause a spectrum of human disease phenotypes associated with upregulated type I interferon signaling. Nat. Genet. 46, 503-509 (2014).

33. Agarwal, A. K. et al. PSMB8 encoding the beta5i proteasome subunit is mutated in joint contractures, muscle atrophy, microcytic anemia, and panniculitisinduced lipodystrophy syndrome. Am. J. Hum. Genet. 87, 866-872 (2010)

34. Liu, Y. et al. Mutations in proteasome subunit beta type 8 cause chronic atypical neutrophilic dermatosis with lipodystrophy and elevated temperature with evidence of genetic and phenotypic heterogeneity. Arthritis Rheum. 64, 895-907 (2012)

35. Watkin, L. B. et al. COPA mutations impair ER-Golgi transport and cause hereditary autoimmune-mediated lung disease and arthritis. Nat. Genet. 47, 654-660 (2015).

36. Volpi, S. et al. Type I interferon pathway activation in COPA syndrome. Clin. Immunol. 187, 33-36 (2018)

37. Harel, T. et al. Recurrent de novo and biallelic variation of ATAD3A, encoding a mitochondrial membrane protein, results in distinct neurological syndromes. Am. J. Hum. Genet. 99, 831-845 (2016).

38. Lepelley, A. et al. Enhanced cGAS-STING-dependent interferon signaling associated with mutations in ATAD3A. J. Exp. Med. 218, e20201560 (2021).

39. Kim, H. et al. Development of a validated interferon score using nanostring technology. J. Interferon Cytokine Res. 38, 171-185 (2018).

40. Mommert, M. et al. Type-I Interferon assessment in 45 minutes using the FilmArray ${ }^{\circledR}$ PCR platform in SARS-CoV-2 and other viral infections. Eur. J. Immunol. 51, 989-994 (2021)

41. Armangue, T. et al. Neonatal detection of Aicardi Goutières syndrome by increased C26:0 lysophosphatidylcholine and interferon signature on newborn screening blood spots. Mol. Genet Metab. 122, 134-139 (2017).

42. Tise, C. G. et al. Aicardi-Goutières syndrome may present with positive newborn screen for X-linked adrenoleukodystrophy. Am. J. Med. Genet. A 185 1848-1853 (2021).

43. Ferreira, A. R., Marques, M. \& Ribeiro, D. Peroxisome and innate immunity: antiviral response and beyond Int. J. Mol. Sci. 20, 3795 (2019)

44. Dixit, E. et al. Peroxisomes are signaling platform for antiviral innate immunity. Cell 141, 668-681 (2010).

45. Gratia, M. et al. Bloom syndrome protein restrains innate immune sensing of micronuclei by cGAS. J. Exp. Med. 216, 1199-1213 (2019)

46. Gul, E. et al. Type I IFN-related NETosis in ataxia telangiectasia and Artemis deficiency. J. Allergy Clin. Immunol. 142, 246-257 (2018)

47. Hartlova, A. et al. DNA damage primes the type I interferon system via the cytosolic DNA sensor STINC to promote anti-microbial innate immun. Immunity 42, 332-343 (2015)

48. Ahmad, S. et al. Breaching self-tolerance to Alu duplex RNA underlies MDA5-mediated inflammation. Cell 172, 797-810 (2018).

49. Sun, L, Wu, J., Du, F Chen, X. \& Chen, Z. J. Cyclic GMP-AMP synthase is a cytosolic DNA sensor that activates the type I interferon pathway. Science 339 , 786-791 (2013)

50. Wu, J. et al. Cyclic GMP-AMP is an endogenous second messenger in innate immune signaling by cytosolic DNA. Science 339, 826-830 (2013).

51. Stetson, D. B. \& Medzhitov, R. Recognition of cytosolic DNA activates an IRF3-dependent innate immune response. Immunity 24, 93-103 (2006).

52. Volkman, H. E., Cambier, S., Gray, E. E. \& Stetson, D. B. Tight nuclear tethering of cGAS is essential for preventing autoreactivity. eLife 8 e47491 (2019).

53. Uggenti, C. et al. cGAS-mediated induction of type I interferon due to inborn errors of histone pre-mRNA processing. Nat. Genet. 52, 1364-1372 (2020). Description of a disturbance of histone stoichiometry in AGS, indicating the immunogenic potential of genomic DNA signalled through cGAS.

54. Mackenzie, K. J. et al. cGAS surveillance of micronucle links genome instability to innate immunity. Nature 548, 461-465 (2017).

55. Zierhut, C. et al. The cytoplasmic DNA sensor cGAS promotes mitotic cell death. Cell 178, 302-315.e23 (2019).

56. Zhao, B. et al. The molecular basis of tight nuclear tethering and inactivation of cGAS. Nature 587 , 673-677 (2020)

57. Pathare, G. R. et al. Structural mechanism of cGAS inhibition by the nucleosome. Nature $587,668-672$ (2020).

58. Boyer, J. A. et al. Structural basis of nucleosomedependent cGAS inhibition. Science 370, 450-454 (2020).

59. Michalski, S et al. Structural basis for sequestration and autoinhibition of cGAS by chromatin. Nature 587 678-682 (2020)

60. Kujirai, T. et al. Structural basis for the inhibition of cCAS by nucleosomes. Science 370, 455-458 (2020).

61. Dhir, A. et al. Mitochondrial double-stranded RNA triggers antiviral signalling in humans. Nature $\mathbf{5 6 0}$, 238-242 (2018)

The first description of type I interferon upregulation in the context of a monogenic 'mitochondrial disease'.

62. Yang, K., Huang, R., Fujihira, H., Suzuki, T. \& Yan, N. N-glycanase NGLY1 regulates mitochondrial homeostasis and inflammation through NRF1. J. Exp. Med. 215, 2600-2616 (2018)

63. Riley, J. S. \& Tait, S. W. Mitochondrial DNA in inflammation and immunity. EMBO Rep. 21, e49799 (2020).

64. Sliter, D. A. et al. Parkin and PINK1 mitigate STING-induced inflammation. Nature 561, 258-262 (2018).

65. Yu, C. H. et al. TDP-43 triggers mitochondrial DNA release via mPTP to activate cGAS/STING in ALS. Cell 183, 636-649 (2020).

66. Kim, J. et al. VDAC oligomers form mitochondrial pores to release mtDNA fragments and promote lupus-like disease. Science 366, 1531-1536 (2019).

67. Fremond, M. L. \& Crow, Y. J. STING-mediated lung inflammation and beyond. J. Clin. Immunol. 41 501-514 (2021).
68. Liu, Y. et al. Activated STING in a vascular and pulmonary syndrome. N. Engl. J. Med. 371, 507-518 (2014).

The first description of gain-of-function mutations in STING in humans.

69. Dobbs, N. et al. STING activation by translocation from the ER is associated with infection and autoinflammatory disease. Cell Host Microbe 18 157-168 (2015).

70. Gonugunta, V. K. et al. Trafficking-mediated STING degradation requires sorting to acidified endolysosomes and can be targeted to enhance anti-tumor response. Cell Rep. 21, 3234-3242 (2017).

71. Gui, X. et al. Autophagy induction via STING trafficking is a primordial function of the cGAS pathway. Nature 567, 262-266 (2019).

72. Lepelley, A. et al. Mutations in COPA lead to abnormal trafficking of STING to the Golgi and interferon signaling. J. Exp. Med. 217, e20200600 (2020).

73. Deng, Z. et al. A defect in COPI-mediated transport of STING causes immune dysregulation in COPA syndrome. J. Exp. Med 217 e20201045 (2020).

74. Mukai, K. et al. Homeostatic regulation of STING by retrograde membrane traffic to the ER. Nat. Commun 12, 61 (2021)

75. Rivara, S. \& Ablasser, A. COPA silences STING. J. Exp. Med 217 e20201517 (2020).

76. Zhang, X. et al. Human intracellular ISG15 prevents interferon-alpha/beta over-amplification and auto-inflammation. Nature 517, 89-93 (2015).

77. Meuwissen, M. E. et al. Human USP18 deficiency underlies type 1 interferonopathy leading to severe pseudo-TORCH syndrome. J. Exp. Med. 213 1163-1174 (2016).

78. Duncan, C. J. A. et al. Severe type I interferonopathy and unrestrained interferon signaling due to a homozygous germline mutation in STAT2. Sci. Immunol. 4, eaav7501 (2019).

The first description of a separation-of-function mutation in STAT2, which results in an upregulation of type I interferon signalling.

79. Gruber, C et al. Homozygous STAT2 gain-of-function mutation by loss of USP 18 activity in a patient with type I interferonopathy. J. Exp. Med. 217, e20192319 (2020).

80. Arimoto, K. I. et al. STAT2 is an essential adaptor in USP18-mediated suppression of type I interferon signaling. Nat. Struct. Mol. Biol. 24, 279-289 (2017).

81. Alsohime, F. et al. JAK inhibitor therapy in a child with inherited USP1 8 deficiency. N. Engl. J. Med. 382 , 256-265 (2020).

82. Kaleviste $\mathrm{E}$ et al Interferon signature in patients with STAT 1 gain-of-function mutation is epigenetically determined. Eur. J. Immunol. 49, 790-800 (2019).

83. Okada, S. et al. Human STAT 1 gain-of-function heterozygous mutations: chronic mucocutaneous candidiasis and type I interferonopathy. J. Clin. Immunol. 40, 1065-1081 (2020).

84. Stellacci, E. et al. The activating p.Ser466Arg change in STAT 1 causes a peculiar phenotype with features of interferonopathies. Clin. Genet. 96, 585-589 (2019).

85. Smyth, A. E. et al. Aortic calcification in a patient with a gain-of-function STAT1 mutation. J. Clin. Immunol. 38, 468-470 (2018)

86. Del Bel, K. L. et al. JAK1 gain-of-function causes an autosomal dominant immune dysregulatory and hypereosinophilic syndrome. J. Allergy Clin. Immunol. 139, 2016-2020 (2017).

87. Gruber, C. N. et al. Complex autoinflammatory syndrome unveils fundamental principles of JAK kinase transcriptional and biochemical function. Immunity 53, 672-684 (2020).

88. Sanchez, G. A., de Jesus, A. A. \& Goldbach-Mansky, R. Monogenic autoinflammatory diseases: disorders of amplified danger sensing and cytokine dysregulation. Rheum. Dis. Clin. North. Am. 39, 701-734 (2013).

89. Ebstein, F., Poli Harlowe, M. C., Studencka-Turski, M. \& Krüger, E. Contribution of the unfolded protein response (UPR) to the pathogenesis of proteasome associated autoinflammatory syndromes (PRAAS). Front. Immunol. 10, 2756 (2019).

90. Brehm, A. et al. Additive loss-of-function proteasome subunit mutations in CANDLE/PRAAS patients promote type I IFN production. J. Clin. Invest. 125 4196-4211 (2015).

91. Frémond, M. L. et al. Overview of STING-associated vasculopathy with inset in infancy (SAVI) among 21 patients. J. Allergy Clin. Immunol. Pract. 9, 803-818 (2021).

92. Briggs, T. A., Paul, A., Rice, G. I. \& Herrick, A. L. RNASEH2B related adult-onset interferonopathy. J. Clin. Immunol. 39, 620-622 (2019). 
93. Abe, J. et al. A nationwide survey of Aicardi-Goutieres syndrome patients identifies a strong association between dominant TREX1 mutations and chilblain lesions: Japanese cohort study. Rheumatology 53 448-458 (2013)

94. V, T. et al. Aicardi-Goutières syndrome due to a paternal mosaic IFIH1 mutation. Neurology. Genet. 6 e384 (2019).

95. Livingston, J. H. et al. A type I interferon signature identifies bilateral striatal necrosis due to mutations in ADAR1. J. Med. Genet. 51, 76-82 (2014).

96. Crowl, J. T., Gray, E. E., Pestal, K., Volkman, H. E \& Stetson, D. B. Intracellular nucleic acid detection in autoimmunity. Annu. Rev. Immunol. 35, 313-336 (2017).

97. Picard, C. et al. Clinical features and outcome of patients with IRAK- 4 and MyD88 deficiency. Medicine 89, 403-425 (2010)

98. Gruber, C. \& Bogunovic, D. Incomplete penetrance in primary immunodeficiency: a skeleton in the closet. Hum. Genet. 139, 745-757 (2020)

99. Gall, A et al Autoimmunity initiates in nonhematopoietic cells and progresses via lymphocytes in an interferon-dependent autoimmune disease. Immunity 36, 120-131 (2012).

100. Gao, D. et al. Activation of cyclic GMP-AMP synthase by self-DNA causes autoimmune diseases. Proc. Natl Acad. Sci. USA 112, 5699-5705 (2015)

101. Gray, E. E., Treuting, P. M., Woodward, J. J. \& Stetson, D. B. Cutting edge: cGAS is required for lethal autoimmune disease in the trex 1-deficient mouse model of Aicardi-Goutieres syndrome. J. Immunol. 195, 1939-1943 (2015)

102. Mackenzie, K. J. et al. Ribonuclease H2 mutations induce a CGAS/STING-dependent innate immune response. EMBO J. 35, 831-844 (2016).

103. Pokatayev, V. et al. RNase $\mathrm{H} 2$ catalytic core AicardiGoutieres syndrome-related mutant invokes cGASSTING innate immune-sensing pathway in mice. J. Exp. Med. 213, 329-336 (2016)

104. Maelfait, J., Bridgeman, A., Benlahrech, A., Cursi, C. ¿ Rehwinkel, J. Restriction by SAMHD1 limits cGAS/STING-dependent innate and adaptive immune responses to HIV-1. Cell Rep. 16, 1492-1501 (2016).

105. Mannion, N. M. et al. The RNA-editing enzyme ADAR1 controls innate immune responses to RNA. Cell. Rep. 9, 1482-1494 (2014).

106. Liddicoat, B. J. et al. RNA editing by ADAR1 prevents MDA5 sensing of endogenous dsRNA as nonself. Science 349, 1115-1120 (2015)

107. Pestal, K. et al. Isoforms of RNA-editing enzyme ADAR 1 independently control nucleic acid sensor MDA5-driven autoimmunity and multi-organ development. Immunity 43, 933-944 (2015).

108. Luksch, H. et al. STING-associated lung disease in mice relies on T cells but not type I interferon. J. Allergy Clin. Immunol. 144, 254-266 (2019).

109. Belot, A. et al. Contribution of rare and predicted pathogenic gene variants to childhood-onset lupus: a large, genetic panel analysis of British and French cohorts. Lancet Rheumatol. 2, 99-109 (2021).

110. Crow, Y. J. Lupus: how much "complexity" is really (just) genetic heterogeneity? Arthritis Rheum. 63, 3661-3664 (2011)

111. Morita, M. et al. Gene-targeted mice lacking the Trex 1 (DNase III) 3' $\rightarrow$ 5' DNA exonuclease develop inflammatory myocarditis. Mol. Cell. Biol. 24 6719-6727 (2004)
112. Maurano, M. et al. PKR and the integrated stress response drive immunopathology caused by ADAR mutation. Immunity 54, 1948-1960.e5 (2021).

113. Chung, $\mathrm{H}$. et al. Human ADAR1 prevents endogenous RNA from triggering translational shutdown. Cell 172 , 811-824 e14 (2018).

114. Goldmann, T. et al. USP18 lack in microglia causes destructive interferonopathy of the mouse brain. EMBO J. 34, 1612-1629 (2015)

115. Michaud, A. P., Bauman, N. M., Burke, D. K., Manaligod, J. M. \& Smith, R. J. Spastic diplegia and other motor disturbances in infants receiving interferon-alpha. Laryngoscope 114, 1231-1236 (2004).

116. Warner, J. D. et al. STING-associated vasculopathy develops independently of IRF3 in mice. J. Exp. Med. 214, 3279-3292 (2017).

117. Bouis, D. et al. Severe combined immunodeficiency in Sting V154M/WT mice. J. Allergy Clin. Immunol. 143 712-725 (2018)

118. Gaidt, M. M. et al. The DNA inflammasome in human myeloid cells is initiated by a STING-cell death program upstream of NLRP3. Cell 171, 1110-1124 (2017).

119. Yamashiro, L. H. et al. Interferon-independent STING signaling promotes resistance to HSV-1 in vivo. Nat. Commun. 11, 3382 (2020).

120. Wu, J., Dobbs, N., Yang, K. \& Yan, N. Interferonindependent activities of mammalian STING mediate antiviral response and tumor immune evasion. Immunity 53, 115-126 (2020).

121. Yum, S., Li, M., Fang, Y. \& Chen, Z. J. TBK1 recruitment to STING activates both IRF3 and NF-kB that mediate immune defense against tumors and viral infections. Proc. Natl Acad. Sci. USA 118, e2100225118 (2021).

122. Kawane, K. et al. Requirement of DNase II for definitive erythropoiesis in the mouse fetal liver. Science 292, 1546-1549 (2001).

123. Yoshida, H., Okabe, Y., Kawane, K., Fukuyama, H. \& Nagata, S. Lethal anemia caused by interferon-beta produced in mouse embryos carrying undigested DNA. Nat. Immunol. 6, 49-56 (2005).

124. Kawane, K. et al. Chronic polyarthritis caused by mammalian DNA that escapes from degradation in macrophages. Nature 443, 998-1002 (2006)

125. Beck-Engeser, G. B., Eilat, D. \& Wabl, M. An autoimmune disease prevented by anti-retroviral drugs. Retrovirology 8, 91 (2011).

126. Rice, G. I. et al. Reverse-transcriptase inhibitors in the Aicardi-Goutières syndrome. N. Engl. J. Med. 379, 2275-2277 (2018).

The first published clinical trial in AGS, indicating an effect of inhibition of reverse transcription on interferon signalling.

127. Rodero, M. P., Fremond, M. L., Rice, G. I., Neven, B. $\&$ Crow, Y. J. JAK inhibition in STING-associated interferonopathy. Ann. Rheum. Dis. 75, e75 (2016).

128. Kothur, K. et al. An open-label trial of JAK 1/2 blockade in progressive IFIH 1 -associated neuroinflammation. Neurology 90, 289-291 (2018).

129. Briand, C. et al. Efficacy of JAK $1 / 2$ inhibition in the treatment of chilblain lupus due to TREX1 deficiency. Ann. Rheum. Dis. 78, 431-433 (2019).

130. Vanderver, A. et al. Janus kinase inhibition in the aicardi-goutières syndrome. N. Engl. J. Med. 383 986-989 (2020)

The largest description published so far of the use of JAK 1 inhibition in AGS

131. Forbes, L. R. et al. Jakinibs for the treatment of immune dysregulation in patients with gain-of-function signal transducer and activator of transcription 1 (STAT1) or STAT3 mutations. J. Allergy Clin. Immunol. 42, 1665-1669 (2018)

132. Sanchez, G. A. M. et al. JAK $1 / 2$ inhibition with baricitinib in the treatment of autoinflammatory interferonopathies. J. Clin. Invest. 128, 3041-3052 (2018).

133. Crow, Y. J., Neven, B. \& Frémond, M. L. JAK inhibition in the type I interferonopathies. J. Allergy Clin. Immunol. https://doi.org/10.1016/j.jaci.2021.07.028 (2021).

134. Neven, B. et al. JAK inhibition in the Aicardi-Goutières syndrome. N. Engl J. Med 383, 2190-2191 (2020).

135. Lee-Kirsch, M. A. et al. Mutations in the gene encoding the 3'-5' DNA exonuclease TREX1 are associated with systemic lupus erythematosus. Nat. Genet. 39, 1065-1067 (2007).

136. Gunther, C. et al. Defective removal of ribonucleotides from DNA promotes systemic autoimmunity. J. Clin. Invest. 125, 413-424 (2015).

137. Crow, Y. J. et al. Characterization of human disease phenotypes associated with mutations in TREX1 RNASEH2A, RNASEH2B, RNASEH2C, SAMHD1, ADAR and IFIH1. Am. J. Med. Genet. 167A, 296-312 (2015).

138. Rice, G. I. et al. Genetic, phenotypic and interferon biomarker status in ADAR 1-related neurological disease. Neuropediatrics 48, 166-184 (2017).

139. Briggs, T. A. et al. Spondyloenchondrodysplasia due to mutations in ACP5: a comprehensive survey. J. Clin. Immunol. 36, 220-234 (2016).

140. Vece, T. J. et al. Copa syndrome: a novel autosomal dominant immune dysregulatory disease. J. Clin. Immunol. 36, 377-387 (2016).

\section{Acknowledgements}

Work contributing to this Review has been funded by the European Research Council (ERC) under the European Union's Horizon 2020 research and innovation programme (grant agreement no. 786142). Y.J.C. also acknowledges a state subsidy managed by the French National Research Agency under the 'Investments for the Future' programme with reference ANR-10-IAHU-01 and the NIHR UK Rare Genetic Disease Research Consortium. Y.J.C. thanks members of the Crow laboratory (in particular M.-L. Fremmond, A. Lepelley, I. Melki, M.-T. el Daher and C. Uggenti) for helpful discussions, C. Uggenti for her generous help with referencing and G. Rice and J. Livingston for their long-standing support. D.B.S. is a Howard Hughes Medical Institute Faculty Scholar and thanks all the members of the Stetson laboratory for insightful discussions over the past 13 years. Finally, the authors sincerely apologize for not being able to cite the many other important articles that have contributed to the development of the type I interferonopathy field.

\section{Author contributions}

The authors contributed equally to all aspects of the article.

Competing interests

The authors declare working as consultants for Related Sciences.

Peer review information

Nature Reviews Immunology thanks the anonymous reviewers for their contribution to the peer review of this work.

\section{Publisher's note}

Springer Nature remains neutral with regard to jurisdictional claims in published maps and institutional affiliations.

(C) Springer Nature Limited 2021 